\title{
Evaluation of three fungal strains for biological removal of Carbamazepine and Atrazine
}

\author{
Gülten Yüksek ${ }^{1,2}$, Lounes Haroune ${ }^{2}$, Didem Okutman Taş ${ }^{1}$, Hubert Cabana ${ }^{2}$ \\ ${ }^{1}$ Istanbul Technical University, Environmental Engineering Department \\ 34469 Maslak, Istanbul, Turkey \\ gulyuksek@itu.edu.tr; okutmand@itu.edu.tr \\ ${ }^{2}$ Groupe de recherche sur l'eau de l'Université de Sherbrooke, Department of Civil Engineering, Université de Sherbrooke \\ 2500 Boulevard de l'Université, Sherbrooke, Québec J1K 2R1, Canada \\ Gulten.Yuksek@Usherbrooke.ca, Lounes.Haroune@Usherbrooke.ca, Hubert.Cabana@Usherbrooke.ca
}

\section{Extended Abstract}

The fate of organic contaminants (OCs) in natural water is not well covered by the world-wide regulation community. The inefficiency of conventional wastewater treatment plants (WWTPs) to remove recalcitrant compounds results in a continuous release of biologically active molecules into the environment. Consequently, new techniques such as biological processes and bioremediation approach by using white rot fungi (WRF) is becoming more popular.

In this study, two recalcitrant model OCs, carbamazepine (CBZ) and atrazine (ATZ) and three different WRF strains (Trametes hirsuta, Coriolopsis polyzona, and Pleurotus dryinus) were selected for the removal of these OCs at three different environmental concentrations. Experimental set up were performed with single and mixed solution of CBZ and ATZ at 100, 150 and $200 \mu \mathrm{g} \mathrm{l}^{-1}$. The sorption mechanisms were evaluated using biocided fungi. The removal of OCs and extracellular enzyme activity (laccase) were quantified by mass spectrometry and UV spectroscopy respectively.

The result showed that the removal efficiency of ATZ and CBZ were up to $85 \%$ even in the presence of biocides, that suggests the high contribution of the membrane biosorption of the WRF on ATZ and CBZ removal from aqueous solutions. The extracellular enzyme activities (i.e. LAC) were not detected in two of the tested fungal stains (ie. $C$. polyzona and $P$. dryinus) but with $T$. hirsuata laccase activity raised up to maximal $600 \mathrm{U} / 1$ after 4 days of cultivation with CBZ $\left(100 \mu \mathrm{g} \mathrm{l}^{-1}\right)$ and $800 \mathrm{U} / 1$ for ATZ $\left(150 \mu \mathrm{g} \mathrm{l}^{-1}\right)$ after 3 days in the single sets experiments. In binary mixed set $(150 \mu \mathrm{g}$ $\left.1^{-1}\right), T$. hirsuta laccase activity reached a maximal level of $900 \mathrm{U} / \mathrm{l}$ after 5 days. ATZ was removed at up to $100 \%$ during the first $24 \mathrm{~h}$ for three WRF studied stains. Interestingly, the ATZ concentration in solution increased after 24h with T. hirsuata suggesting a passive uptake and extracellular release, while the living and biocided fungi had the same behaviour. Also, the removal efficiency of $\mathrm{CBZ}$ at three environmental concentrations was up to $100 \%$ on the first $24 \mathrm{~h}$ without any fluctuation in the removal rate over a 5 day incubation period.

\section{Acknowledgements}

This study was funded by the Scientific and Technological Research Council of Turkey (TUBITAK) as well as the Natural Science and Engineering Research Council of Canada (NSERC) grant programs. Also the authors would want to thank Olivier Savary and Sabrina Saibi for their technical support. 